\title{
Surface characterisation of synthetic coal chars made from model compounds
}

A. Arenillas ${ }^{\mathrm{a}}$, C. Pevida ${ }^{\mathrm{a}}$, F. Rubiera ${ }^{\mathrm{a}}$, J.M. Palacios ${ }^{\mathrm{b}}$, R. Navarrete ${ }^{\mathrm{c}}$, R. Denoyel $^{\mathrm{c}}$, J. Rouquerol ${ }^{\mathrm{c}}$ and J.J. Pis ${ }^{\mathrm{a}}$

${ }^{a}$ Instituto Nacional del Carbón, C.S.I.C., Apartado 73, 33080, Oviedo, Spain

binstituto de Catálisis y Petroleoquímica, C.S.I.C. Campus UAM-Cantoblanco, 28049 Madrid, Spain

'MADIREL, C.N.R.S. Université de Provence, Centre de Saint Jérôme, 13397 Marseille, Cedex 20, France

\begin{abstract}
Knowledge of surface properties is essential for understanding the reaction mechanisms involved in several coal conversion processes. However, due to the complexity and heterogeneity of coal this is rather difficult and the use of known model compounds could be a valuable tool. Single model compounds have been widely used, but they give a quite simplified picture. In this work a mixture of model compounds in a phenol-formaldehyde matrix was cured in order to create cross-linked structures. The obtained synthetic coal was pyrolysed in a fixed bed reactor, under helium atmosphere. The surface composition of the chars was evaluated by XPS, adsorption gravimetry of water vapour, temperatureprogrammed desorption and potentiometric titration. Texture was characterised by $\mathrm{N}_{2}$ and $\mathrm{CO}_{2}$ adsorption isotherms at $77 \mathrm{~K}$ and $273 \mathrm{~K}$, respectively, and immersion calorimetry in benzene. The results obtained from the different techniques were contrasted in order to give an overview of the surface properties (chemical and physical) of the samples studied. Chars obtained under the same operating conditions from a high volatile bituminous coal were used as a reference.
\end{abstract}

Keywords: A. Carbon precursor; B. Carbonization, Mixing; D. Texture, Chemical structure.

\section{INTRODUCTION}

Coal chemical and physical structure is extremely complex and heterogeneous, and consists of a highly cross-linked network. Knowledge of the surface properties is essential for understanding and controlling the reactions involved in several coal conversion processes. Therefore, several approaches have been applied for a better knowledge of coal structure. Thus, single model compounds have been extensively used to simulate coal behaviour $[1,2]$. 
Although interesting results have been obtained in this way, the picture is a simplified one and some important aspects such as interactions and cross-linking reactions are omitted in the case of single compounds. The use of a mixture of model compounds with known functionalities, in an organic matrix similar to that of coal, may be a more reasonable approach in order to overcome some of the difficulties encountered in the study of the mechanisms involved in coal conversion processes [3]. Using this kind of mixture the chemical environment is welldefined and understanding of the ensuing mechanisms is simplified. Furthermore, the absence of mineral matter in the mixture avoids possible catalytic effects, undesirable when trying to isolate the effect of carbon active surface groups.

The evolution of nitrogen during coal processing is a matter of concern due to environmental problems. Single model compounds containing nitrogen or special surface treatments like ammoxidation are widely used in order to elucidate the involved pathways. However, for a more accurate study of the reaction mechanisms involving nitrogen, this must be distributed throughout the bulk of the coal, not just on the surface, and with known functionalities.

In this work synthetic carbon materials were obtained from model compounds with nitrogen functionalities commonly present in coal, in order to be subsequently used as reference samples which, following homogeneous precursor mixing and curing, provide adequate representations of coal char composition and structure. Provided their stability or their decomposition products can be adjusted as needed, prior knowledge of the functionalities present in these synthetic carbon materials are expected to make it easier to elucidate the mechanisms involved in heterogeneous reactions, such as the NO-C reaction. Chars obtained from a high volatile bituminous coal were also chemically and physically characterised and used as a reference.

\section{EXPERIMENTAL}

A mixture of model compounds was prepared using phenol-formaldehyde resin (PFR) as the carbonaceous matrix, 3,4,9,10-perilentetracarboxylic dianhydride (PTC), and nitrogencontaining model compounds, polyvinylpyrrolidone (PVP) and poly-4-vinylpyridine (p4VP). PFR is a simplified model of the aromatic structure in a bituminous coal and PTC introduces carboxyl and anhydride groups in that carbonaceous matrix. Most of nitrogen present in coal is bound into its organic carbonaceous part, thus PVP was used to introduce nitrogen and oxygen in five member aromatic rings and $\mathrm{p} 4 \mathrm{VP}$ as a source of pyridinic nitrogen.

This mixture was cured to favour rearrangements and cross-linkages between the components, at optimised conditions ( $5 \mathrm{~K} \mathrm{~min}^{-1}$ up to $698 \mathrm{~K}$ under Ar flow) and denoted as synthetic coal 
(SC). Optimisation of SC preparation was made in order to obtain a thermal behaviour and chemical composition similar to those of a high volatile bituminous coal, CA, chosen as reference material. CA and SC were used as the source of the carbonaceous materials (i.e. chars) studied in this work.

The samples SC and CA were prepared for solid FTIR analysis following the standard procedure. A sample:KBr mixture (1:100 ratio) was ground with a mortar and pestle. Pellets were then pressed and dried at $373 \mathrm{~K}$ overnight. All absorbance spectra were recorded on a Nicolet Magna IR-560 spectrometer by co-adding 100 interferograms obtained at a resolution of $4 \mathrm{~cm}^{-1}$. Each spectrum was corrected for scattering using two baselines (3800-1800 and $1800-400 \mathrm{~cm}^{-1}$ ) and was normalised to $1 \mathrm{mg}(\mathrm{daf}) \mathrm{cm}^{-2}$.

SC and CA were pyrolysed in a fixed bed reactor under He flow at $15 \mathrm{~K} \mathrm{~min}^{-1}$ up to different temperatures (873 and $1123 \mathrm{~K}$ ) with a soaking time of 5 minutes. The chars obtained were denoted as SC873, CA873, SC1123 and CA1123.

For surface characterisation X-ray photoelectron spectra (XPS) were obtained in a VG ESCALAB 200R spectrometer. The spectrometer was fitted with a hemispherical electron analyser and an X-ray source of $\mathrm{Mg}(\mathrm{K} \alpha=1253.6 \mathrm{eV})$ operating at $6.7 \times 10^{-7} \mathrm{~Pa}$. The spectra were achieved at a constant energy mode of $10 \mathrm{eV}$ in order to get a spectral resolution of \pm 0.2 $\mathrm{eV}$. To correct the effects of sample charging, the C1s peak with a binding energy (BE) of $284.5 \mathrm{eV}$ was used as reference.

The water adsorption isotherms were carried out at $298 \mathrm{~K}$, by means of a quasi-equilibrium procedure [4], where the adsorptive is continuously introduced at a rate low enough to ensure the quasi-equilibrium conditions, whereas the quasi-equilibrium pressure and the corresponding amount adsorbed were continuously recorded. About $50 \mathrm{mg}$ of each sample were degassed in situ at $393 \mathrm{~K}$ under vacuum until constant weight was achieved. In the work reported here it took between 48 and 72 hours, depending on the sample, to carry out the full adsorption-desorption isotherm. The authors' experience is that this time duration is normally sufficient to ensure quasi-equilibrium [5]. The water isotherms were fitted to the DubininSerpinsky (DS) equation to calculate $\mathrm{a}_{0}$, value indicative of the number of primary adsorption sites [6].

Temperature-programmed desorption (TPD) tests were carried out in a thermogravimetric analyser, TGA, linked to a Fourier transform infrared (FTIR) spectrometer. In these experiments the temperature was raised to $1123 \mathrm{~K}_{\text {at }} 15 \mathrm{~K} \mathrm{~min}^{-1}$, under $50 \mathrm{~mL} \mathrm{~min}^{-1}$ of argon. Acidic and basic site concentrations were determined following the Boehm titration method $[7,8]$. Weighed amounts of the samples $(1 \mathrm{~g})$ were placed in $50 \mathrm{~mL}$ of either $0.05 \mathrm{~N} \mathrm{NaHCO}$, 
$\mathrm{Na}_{2} \mathrm{CO}_{3}, \mathrm{NaOH}$ solutions, sealed and allowed to equilibrate with gentle agitation for at least 2 days at $298 \mathrm{~K}$. Aliquots of the solutions were then taken, acidified with excess $\mathrm{HCl} 0.05 \mathrm{~N}$ and titrated with standard $0.05 \mathrm{~N} \mathrm{NaOH}$.

The point of zero charge (PZC) was also evaluated. Due to the low amount of sample (0.5 g) the reverse mass titration method was employed [9, 10]. The samples were diluted in water and shaken under inert atmosphere $\left(\mathrm{N}_{2}\right)$ at ambient temperature (298 K) for two days. Then the $\mathrm{pH}$ was measured and fresh water was added. The concentrations of solid varied between 20 and $1 \mathrm{wt} \%$.

Textural characterisation of the samples was performed by $\mathrm{N}_{2}(77 \mathrm{~K})$ and $\mathrm{CO}_{2}(273 \mathrm{~K})$ adsorption isotherms. The apparent surface areas were obtained by using the BET equation in its linear form [11] in the relative pressure range of 0.01-0.07, where the BET plots show linearity. The micropore size distribution was analysed by means of the DubininRadushkevich (DR) method [12].

The enthalpies of immersion of the samples into $\mathrm{C}_{6} \mathrm{H}_{6}$ were determined at $298 \mathrm{~K}$ with a TianCalvet differential microcalorimeter. Prior to the experiments, the samples ( $100 \mathrm{mg}$ ) were degassed under vacuum at $393 \mathrm{~K}$.

\section{RESULTS AND DISCUSSION}

The chemical analyses of the samples studied are presented in Table 1. A reasonable resemblance between some of the features of the parent samples (i.e. SC and CA) can be observed. The main differences are the absence of ash and sulphur in SC, as no mineral matter or sulphur compounds were included in its composition.

Figure 1 shows the FTIR spectrum of SC. It can be seen that it is not the sum of the individual components (i.e. model compounds). The curing step has produced links and condensation reactions, producing as a result a unique structure. Using the bituminous coal as a reference, it can be appreciated that both spectra are quite similar. The main differences are due to the higher content of $\mathrm{C}=\mathrm{O}$ groups $\left(1700 \mathrm{~cm}^{-1}\right.$ band $)$ in accordance with the higher oxygen content (see Table 1) and the absence of mineral matter (1100-1000 $\mathrm{cm}^{-1}$ bands) in SC. Sample SC also appears to have a lower content of aliphatic C-H groups $\left(2900 \mathrm{~cm}^{-1}\right.$ band).

The obtention of a synthetic coal that resembles well the overall composition and structure of coal, including nitrogen functionalities, is a difficult task. Determination of the nitrogen functionalities in both materials, SC and CA, by XPS according to the assignation of Stanczyck et al [13], are given in Table 2. 
Taking into account the complexity and heterogeneity of coal, it can be said that the obtained synthetic coal provides a reasonable representation of the composition (even nitrogen functionalities) of the bituminous coal taken as a reference material.

The adsorption of polar vapours (i.e. water) occurs at specific sites and is characterised by relatively weak interactions with respect to the adsorption of non-polar vapours. For pure carbons exposed to air, the polar sites are chemisorbed oxygen molecules. In the case of the samples used in this work heteroatoms such as oxygen or nitrogen for SC samples, and also sulphur in the case of CA, may act as adsorption sites. Water vapour is probably the polar adsorptive most frequently used. In this case, the interaction is primarily by hydrogen bonding giving heat of adsorption values in the range $5-15 \mathrm{~kJ} \mathrm{~mol}^{-1}$ [14]. The water adsorption isotherms of the samples are presented in Figure 2. The shape of the isotherms indicates that all the samples are weakly polar materials, as expected. However, the water adsorption of SC samples is higher than those of CA. This indicates that SC samples have a higher concentration of primary adsorption sites, in agreement with the higher content of heteroatoms, particularly oxygen in $\mathrm{C}=\mathrm{O}$ groups, in the parent coal, SC (see Figure 1).

Analysis of the initial region of water isotherms, gives different parameters such as $a_{0}$ (measure of the polarity of the surface), $\mathrm{a}_{\mathrm{s}}$ (the limiting amount of water adsorbed as $\mathrm{p} / \mathrm{p}^{0}$ approaches unity) and c (ratio between the overall rates of adsorption and desorption) [6].

The parameters resulting from application of the DS equation to the chars are shown in Table 3. As expected, chars SC873 and CA873 presented higher concentrations of primary adsorption centres, $a_{0}$, than SC1123 and CA1123. Notwithstanding the relative enrichments of sample CA in inorganic matter upon heat treatments (Table 1), this confirms that as the treatment temperature increases the surface polarity decreases and, as a consequence, the surfaces become more hydrophobic by the loss of oxygen surface groups. It is worth noting that, although CA chars have sulphur and mineral matter in their composition, which are not present in SC chars, the oxygen content seems to be more important, giving for SC samples higher concentration of adsorption sites (i.e. higher $\mathrm{a}_{0}$ and $\mathrm{a}_{\mathrm{s}}$ values).

Figure 3 shows the $\mathrm{CO}$ and $\mathrm{CO}_{2}$ profiles followed by FTIR analysis, during the TPD tests. A detailed quantitative analysis has not been conducted in this work. The sensitivity of FTIR to $\mathrm{CO}_{2}$ is much higher than to $\mathrm{CO}$, due to the low absorption coefficient of $\mathrm{CO}$. It is generally assumed that each type of oxygen surface group decomposes to a specific product, e.g. $\mathrm{CO}_{2}$ derives from carboxyl and lactone groups, and CO from carbonyl and hydroxyl groups and ether-type oxygen. 
As expected, the profiles showed that $\mathrm{CO}$ and $\mathrm{CO}_{2}$ desorption is greater for the chars obtained at $873 \mathrm{~K}$ (SC873 and CA873) than for the chars obtained at $1123 \mathrm{~K}$, confirming that the lower temperature chars have a higher oxygen concentration on their surface. The CO evolution of the low and high temperature chars was remarkably different, and relatively little CO was evolved from the high temperature chars. This implies that the oxygen contained in carbonyl, hydroxyl and ether groups decreases markedly during the heat treatment at $1123 \mathrm{~K}$. These groups would constitute a main source of primary centres for water adsorption on the chars. This could be the reason for the differences in $a_{0}$ between the chars obtained at 873 and $1123 \mathrm{~K}$.

In the case of the $\mathrm{CO}_{2}$ evolution profiles shown in Figure 3, the differences are greater in the case of CA1123. Nevertheless, in both cases there is a decrease in the carboxyl groups concentration with the heat treatment temperature, as expected.

The acidic site concentrations are negligible indicating that the coals and their chars are basic carbons. Basic sites concentration obtained by titration of the remaining $\mathrm{HCl}$ in solution, with $\mathrm{NaOH} 0.05 \mathrm{~N}_{\text {gave }} 0.68 \mathrm{meq} \mathrm{g}^{-1}$ and $0.57 \mathrm{meq} \mathrm{g}^{-1}$ for CA1123 and SC1123, respectively.

The point of zero charge, PZC, was also evaluated for the SC and CA series by the reverse mass titration method. The principle of this method is measuring $\mathrm{pH}$ as a function of mass concentration of a solid sample. Theoretically the $\mathrm{pH}$ gradually approaches to a constant value and for pure samples, in the absence of specific adsorption, this value corresponds to the $\mathrm{pH}_{\text {PZC }}$. Table 4 shows these values for the samples studied, which clearly indicate that they are basic carbons.

According to the $\mathrm{N}_{2}$ adsorption isotherms SC and CA appear to be non-porous solids, as they present a negligible adsorbed volume. However, pyrolysis develops porosity in the chars with a corresponding increase in $\mathrm{N}_{2}$ adsorption. Analysis of the $\mathrm{N}_{2}$ adsorption isotherms by the BET and DR methods results in the textural parameters summarized in Table 5.

BET surface areas are higher for the SC chars (SC873 and SC1123) than for the CA chars obtained at the same temperature (CA873 and CA1123). This is consistent with the higher volatile matter content of SC-based samples (Table 1). The parameters calculated by the DR equation suggest that the chars develop a small amount of microporosity during the heat treatment. In the case of SC1123 the similarity between the micropore surface area, Smic= 2 Wo/L, and the apparent BET surface area, besides the comparatively low value of its average micropore width, $\mathrm{L}$, indicate that this carbonaceous material is mainly microporous.

$\mathrm{CO}_{2}$ adsorption at room temperature $(293 \mathrm{~K})$ is a useful tool for the study of the microporosity of the samples. The textural parameters calculated through the application of the DR equation 
to $\mathrm{CO}_{2}$ adsorption data are shown in Table 6. The differences in micropore volume, adsorption energy and micropore surface area between the chars from the same parent coal (SC or CA) seem negligible in comparison to the DR parameters.

The data from $\mathrm{N}_{2}$ and $\mathrm{CO}_{2}$ adsorption experiments must be used in a complementary way in order to fully characterise micro and mesoporosity. In this respect the values of the $\mathrm{N}_{2} / \mathrm{CO}_{2}$ surface area ratios $<1$, suggest that all the samples contain narrow micropores [15]. Heat treatment opened up some previously inaccessible narrow micropores, and/or selectively destroyed some of them. SC provided chars with less narrow microporosity, and hence a more open structure, than CA. From the data of Tables 5 and 6 it can be inferred that the samples containing the most micropores (SC chars) are also the ones with a more open microstructure. On the other hand, it is possible to obtain surface area values from immersion calorimetry experiments assuming that there is a direct relationship between the energy of immersion and the total surface area of a microporous material [16]. Table 7 shows the experimental enthalpies of immersion in $\mathrm{C}_{6} \mathrm{H}_{6}$ and the surface areas for the SC1123 and CA1123 samples. In the other samples a swelling effect was observed, and the method is partly invalidated as there are energetic components different from a simple adsorption process.

It can be seen in Table 7 that equal surface area values were obtained for SC1123 by immersion in benzene and the BET method, while for the sample with smaller micropores, CA1123, the surface area calculated from benzene immersion is higher than the corresponding BET value. In very narrow micropores, the BET method underestimates the surface area of the pores [17]. It is also worth noting that benzene has a similar molecular size $(0.37 \mathrm{~nm})$ to that of $\mathrm{N}_{2}(0.36 \mathrm{~nm})$.

\section{CONCLUSIONS}

The synthetic coal SC obtained from model compounds and its chars represent quite well the composition and functionalities of a reference coal and its chars. However, synthetic coal chars develop higher textural properties. It is proposed that the synthetic carbon materials could be of great interest for elucidating mechanisms of heterogeneous reactions, due to the presence of active sites similar to those found in coal char but with more accessibility.

\section{REFERENCES}

[1] Pels JR, Kapteijn F, Moulijn JA, Zhu Q, Thomas KM. Evolution of nitrogen functionalities in carbonaceous materials during pyrolysis. Carbon 1995; 33(11):1641-1653. 
[2] Murata S, Nakamura M, Miura M, Nomura M. Pyrolysis of coal model compounds. Thermal behavior of benzyl-substituted polyaromatic compounds. Energy \& Fuels 1995; 9(5):849-854.

[3] Arenillas A, Pevida C, Rubiera F, García R, Pis JJ. Characterisation of model compounds and a synthetic coal by TG/MS/FTIR to represent the pyrolysis behaviour of coal. J Anal Applied Pyrol (In Press).

[4] Rouquerol J, Davy L. Automatic gravimetric apparatus for recording the adsorption isotherms of gases or vapours onto solids. Thermochimica Acta 1978; 24(2):391-397.

[5] Poirier JE, Francois M, Cases J-M, Rouquerol J. Study of water adsorption on Namontmorillonite: new data owing to the use of a continuous procedure. Proceedings of the 2nd Engineering Foundation Conference on Fundamentals of Adsorption 1987:473.

[6] Dubinin MM, Serpinsky VV. Isotherm equation for water vapor adsorption by microporous carbonaceous adsorbents. Carbon 1981; 19(5):402-403.

[7] Boehm HP. Some aspects of the surface chemistry of carbon blacks and other carbons. Carbon 1994; 32(5):759-769.

[8] Boehm HP. Chemical identification of surface groups. In: Eley DD, Pines H, Weisz PB, editors. Advances in Catalysis, Academic Press, New York, 1966; 16:179.

[9] Noh JS, Schwarz JA. Estimation of the point of zero charge of simple oxides by mass titration. J Colloid Interf Sci 1989; 130(1):157-164.

[10] Zalac S, Kallay, N. Application of mass titration to the point of zero charge determination. J Colloid Interf Sci 1992; 149(1):233-240.

[11] Parra JB, de Sousa JC, Bansal RC, Pis JJ, Pajares JA. Characterization of activated carbons by the BET equation-an alternative approach. Adsorption Sci Technol 1995; 12:5166.

[12] Byrne JF, Marsh H. Introductory overview. In: Patrick JW, editor. Porosity in carbons: Characterization and applications. Edward Arnold, 1995; 1-48.

[13] Stanczyck K, Dziembaj R, Piwowarska Z, Witkowski S. Transformation of nitrogen structures in carbonization of model compounds determined by XPS. Carbon 1995; 33(10):1383-1392.

[14] Bradley RH. Polar interactions at carbon surfaces described by the Dubinin equation and by an XPS-based model. Adsorption Sci Technol 1997; 15:477-484.

[15] Rodríguez-Reinoso F, Linares-Solano A. Microporous structure of activated carbons as revealed by adsorption methods. In: Thrower PA, editor. Chemistry and Physics of Carbons, Marcel Dekker, New York, 1989; 21:1-146. 
[16] Denoyel R, Fernández-Colinas F, Grillet Y, Rouquerol J. Polar interactions at carbon surfaces described by the Dubinin equation and by an XPS-based model. Langmuir 1993; 9:515-518.

[17] Rodríguez-Reinoso F, Molina-Sabio M. Textural and chemical characterisation of microporous carbons. Adv Colloid Interf Sci 1998; 76-77:271-294. 


\section{LIST OF TABLES}

Table 1. Proximate and ultimate analyses of the coals and their chars

Table 2. Nitrogen functionalities distribution in SC and CA according to XPS analysis

Table 3. Dubinin-Serpinsky parameters calculated from the water adsorption isotherms at $298 \mathrm{~K}$

Table 4. Values of $\mathrm{pH}_{\mathrm{PZC}}$ for the samples studied

Table 5. Textural parameters derived from $\mathrm{N}_{2}$ adsorption isotherms at $77 \mathrm{~K}$

Table 6. Textural parameters derived from $\mathrm{CO}_{2}$ adsorption data at $273 \mathrm{~K}$ and $\mathrm{N}_{2} / \mathrm{CO}_{2}$ surface area ratios

Table 7. Enthalpies of immersion at $298 \mathrm{~K}$ in $\mathrm{C}_{6} \mathrm{H}_{6}$ and surface areas calculated for the samples studied

\section{LIST OF FIGURES}

Fig.1. FTIR spectra of the synthetic coal (SC) and the bituminous coal (CA)

Fig. 2. Isotherms of water adsorption at $298 \mathrm{~K}$ for coals SC, CA and their chars

Fig. 3. $\mathrm{CO}$ and $\mathrm{CO}_{2}$ evolution during TPD tests, followed by FTIR 
Table 1. Proximate and ultimate analyses of the coals and their chars

\begin{tabular}{|c|c|c|c|c|c|c|c|}
\hline & \multicolumn{2}{|c|}{ Proximate Analysis $\left(\mathrm{wt} \%, \mathrm{db}^{\mathrm{a}}\right)$} & \multicolumn{5}{|c|}{ Ultimate Analysis (wt\%, daf ${ }^{b}$ ) } \\
\hline & $\mathrm{VM}$ & Ash & $\mathrm{C}$ & $\mathrm{H}$ & $\mathrm{N}$ & $S$ & $\mathrm{O}$ \\
\hline SC & 39.2 & 0.0 & 81.8 & 5.4 & 2.6 & 0.0 & 10.2 \\
\hline CA & 37.7 & 7.6 & 84.4 & 5.5 & 1.8 & 1.6 & 6.7 \\
\hline SC873 & 19.1 & 0.0 & 89.7 & 2.4 & 2.2 & 0.0 & 5.7 \\
\hline SC1123 & 11.3 & 0.0 & 95.5 & 1.2 & 1.6 & 0.0 & 1.7 \\
\hline CA873 & 16.2 & 11.3 & 89.3 & 2.6 & 2.2 & 1.6 & 4.3 \\
\hline CA1123 & 7.9 & 14.2 & 93.1 & 1.3 & 2.0 & 2.1 & 1.5 \\
\hline
\end{tabular}

${ }^{\text {adry basis; }}{ }^{\text {b dry ash free basis }}$ 
Table 2. Nitrogen functionalities distribution in SC and CA according to XPS analysis

\begin{tabular}{cccc}
\hline & N pyridinic (\%) & N pyrrolic (\%) & N quaternary (\%) \\
\hline SC & 38 & 52 & 10 \\
SC1123 & 44 & 45 & 11 \\
CA & 36 & 50 & 14 \\
CA1123 & 41 & 42 & 17 \\
\hline
\end{tabular}


Table 3. Dubinin-Serpinsky parameters calculated from the water adsorption isotherms at $298 \mathrm{~K}$

\begin{tabular}{cccc}
\hline & \multicolumn{3}{c}{ DS equation } \\
\cline { 2 - 4 } & $\mathrm{a}_{\mathrm{o}}\left(\mathrm{mmol} \mathrm{g}^{-1}\right)$ & $\mathrm{c}$ & $\mathrm{a}_{\mathrm{s}}\left(\mathrm{mmol} \mathrm{g}^{-1}\right)$ \\
\hline SC873 & 2.3 & 1.1 & 7.0 \\
SC1123 & 1.5 & 9.3 & 10.8 \\
CA873 & 1.3 & 0.7 & 3.0 \\
CA1123 & 0.2 & 3.0 & 3.8 \\
\hline
\end{tabular}


Table 4. Values of $\mathrm{pH}_{\mathrm{PZC}}$ for the samples studied

\begin{tabular}{cc}
\hline Sample & $\mathrm{pH}_{\mathrm{PZC}}$ \\
\hline SC & 8.2 \\
SC873 & 9.8 \\
SC1123 & 10.8 \\
CA & 7.4 \\
CA873 & 8.3 \\
CA1123 & 12.1 \\
\hline
\end{tabular}


Table 5. Textural parameters derived from $\mathrm{N}_{2}$ adsorption isotherms at $77 \mathrm{~K}$

\begin{tabular}{cccccc}
\hline & $\mathrm{S}_{\text {BET }}\left(\mathrm{m}^{2} \mathrm{~g}^{-1}\right)$ & \multicolumn{5}{c}{ DR method } \\
\cline { 3 - 6 } & & Wo $\left(\mathrm{mm}^{3} \mathrm{~g}^{-1}\right)$ & Eo $\left(\mathrm{kJmol}^{-1}\right)$ & $\mathrm{L}(\mathrm{nm})$ & Smic $\left(\mathrm{m}^{2} \mathrm{~g}^{-1}\right)$ \\
\hline $\mathrm{SC}$ & 0.2 & - & - & - & - \\
$\mathrm{SC} 873$ & 36 & 4 & 16 & 2.5 & 3 \\
$\mathrm{SC} 1123$ & 87 & 34 & 25 & 0.8 & 85 \\
$\mathrm{CA}$ & 2 & - & - & - & - \\
CA873 & 9 & 2 & 17 & 1.9 & 3 \\
CA1123 & 32 & 7 & 18 & 1.6 & 8 \\
\hline
\end{tabular}


Table 6. Textural parameters derived from $\mathrm{CO}_{2}$ adsorption data at $273 \mathrm{~K}$ and $\mathrm{N}_{2} / \mathrm{CO}_{2}$ surface area ratios

\begin{tabular}{cccccc}
\hline & \multicolumn{2}{c}{ DR method } & $\begin{array}{c}\mathrm{N}_{2} / \mathrm{CO}_{2} \text { Surface } \\
\text { Area Ratio }\end{array}$ \\
\cline { 2 - 5 } & Wo $\left(\mathrm{mm}^{3} \mathrm{~g}^{-1}\right)$ & ${\mathrm{Eo}\left(\mathrm{kJmol}^{-1}\right)}^{\mathrm{L}(\mathrm{nm})}$ & $\left.\mathrm{Smic}^{2} \mathrm{~g}^{-1}\right)$ & 82 & 0.002 \\
$\mathrm{SC}$ & 46 & 21 & 1.1 & 257 & 0.140 \\
$\mathrm{SC873}$ & 116 & 23 & 0.9 & 232 & 0.375 \\
SC1123 & 123 & 22 & 1.1 & 82 & 0.024 \\
CA & 48 & 21 & 1.6 & 171 & 0.053 \\
CA873 & 87 & 22 & 1.0 & 212 & 0.151 \\
CA1123 & 87 & 25 & 0.8 & & \\
\hline
\end{tabular}


Table 7. Enthalpies of immersion at $298 \mathrm{~K}$ in $\mathrm{C}_{6} \mathrm{H}_{6}$ and surface areas calculated for the samples studied

\begin{tabular}{ccc}
\hline & Immersion enthalpy $\left(\mathrm{J} \mathrm{g}^{-1}\right)$ & Surface area $\left(\mathrm{m}^{2} \mathrm{~g}^{-1}\right)$ \\
\hline SC1123 & 9.2 & 83 \\
CA1123 & 6.2 & 56 \\
\hline
\end{tabular}




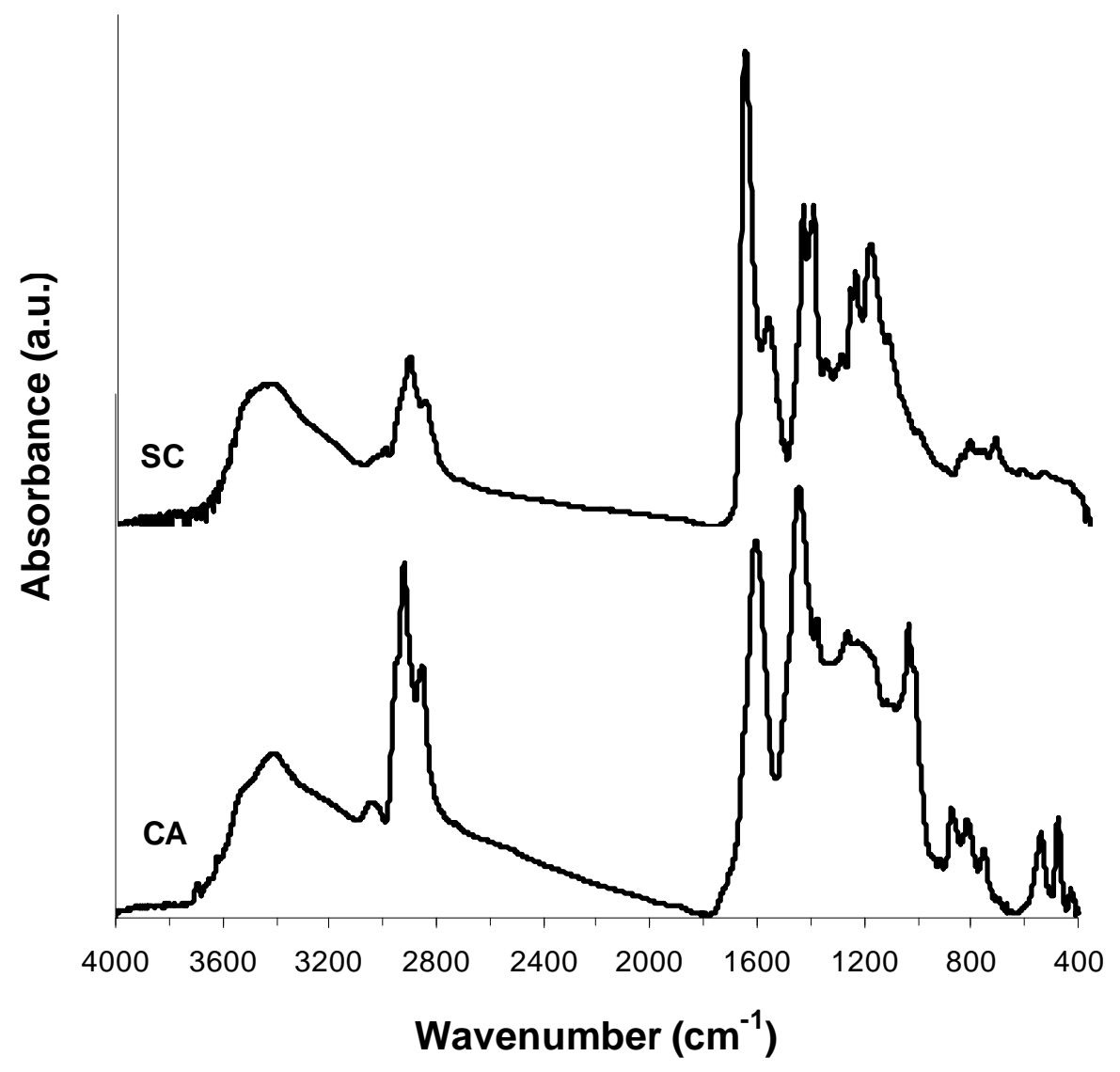

Figure 1 

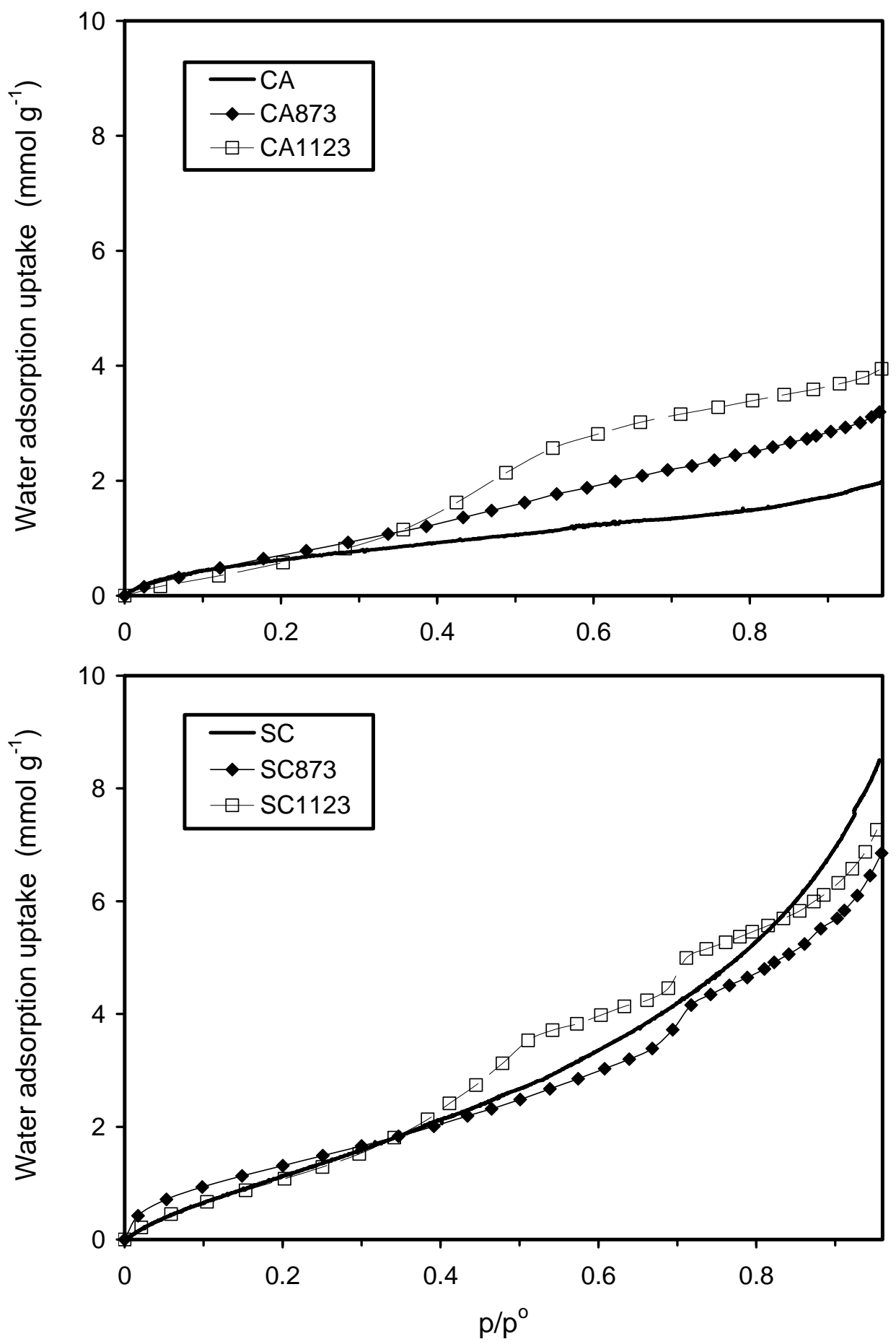

Figure 2 

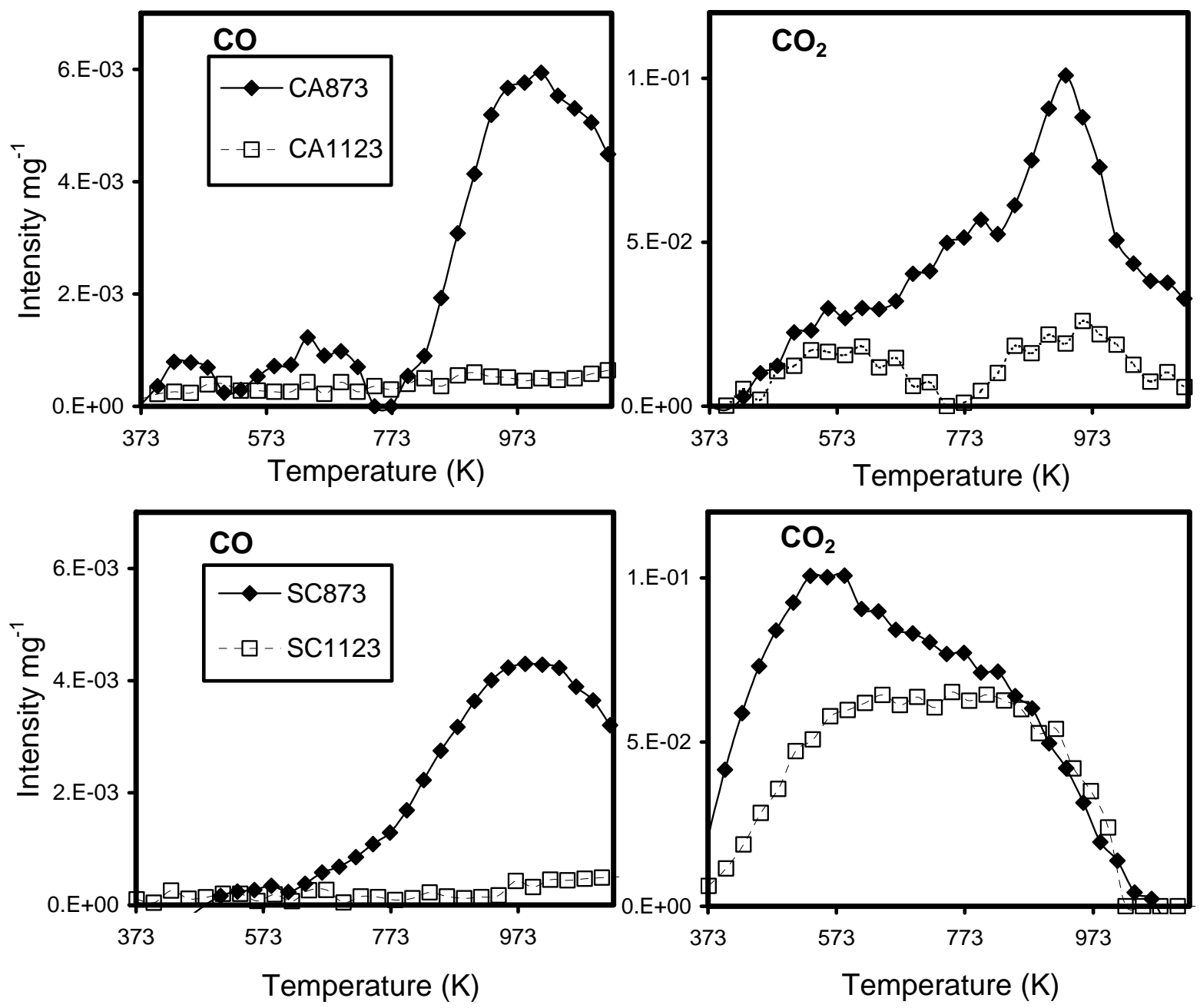

Figure 3 